\title{
Singular Value Decomposition Aided Cubature Kalman Filter with Neural Network in Uncalibrated Binocular Stereo Visual Servoing System
}

\author{
Wei Zhao ${ }^{1,2}$, Huiguang $\mathrm{Li}^{1}$, Liying Zou ${ }^{1}$ and Wenjuan Huang ${ }^{3}$ \\ ${ }^{1}$ School of Electrical Engineering, Yanshan University, No. 438, Hebei Street, \\ Qinhuangdao 066004, P. R. China \\ ${ }^{2}$ Qinggong College, North China University of Science and Technology, No.11, \\ Daxue west road, Tangshan, 063000, P. R. China \\ ${ }^{3}$ Tangshan Vocational and Technical College, No. 120, Xinhua west road, \\ Tangshan, 063004, P. R. China \\ ${ }^{1}$ zhwei19800@163.com, ${ }^{2}$ ysulihuiguang@163.com, ${ }^{3}$ zouliying2007@126.com, \\ ${ }^{4}$ huangwenjuan-2007@163.com
}

\begin{abstract}
In this paper, a method for estimating the image Jacobi matrix in an uncalibrated binocular stereo visual servoing system is presented. The method is based on the singular value decomposition aided Cubature Kalman filter with neural network (NNSVDCKF). First of all, the parameter expression of image Jacobi matrix is obtained by analysis of model of eye-in-hand binocular vision. Then, in the case of the unknown camera parameters, the singular value decomposition aided Cubature Kalman filter (SVDCKF) algorithm is used to estimate the Jacobi matrix, and the neural network (NN) as the noise compensator which compensates the process noise and measurement noise. To demonstrate the effectiveness and practicality of the proposed algorithm, an uncalibrated binocular visual servoing control system based on 6DOF robot PUMA560 is established. Finally, the three algorithms of NNSVDCKF, SVDCKF, and standard Kalman filter (KF) are compared in the uncalibrated servoing system. The simulation results show that the NNSVDCKF algorithm improves the dynamic performance of the system greatly, and has robustness to the constraint in field-of-view (FOV) of the camera.
\end{abstract}

Keywords: Uncalibrated servoing system; Binocular Stereo; Visual Servo; Cubature Kalman filter; Neural network

\section{Introduction}

Computer visual servoing control is to obtain and analyze the image information, extracts the visual information as a feedback signal, and then controls the position and pose of the end-effecter of robot in a closed-loop [1,2]. According to different positions of installed camera, the visual servoing control system is divided into eye-in-hand system and eye-to-hand system. Also, it can be divided into position-based visual servoing (PBVS), image-based visual servoing (IBVS), and their hybrid visual servoing [3, 4]. Among these, IBVS has attracted more and more attention for scholars in recent years, because of the direct control of the two-dimensional image without requirement to consider the three-dimensional reconstruction of the object model, as well as the strong robustness to calibration of internal and external camera parameters [5].

When designing IBVS system, the image Jacobi matrix is needed which describe the relationship between the motion of the end-effecter in the robot space and the motion of image feature in the image space. And the accuracy of the servoing system is determined by the accuracy of image Jacobi matrix [6]. Therefore, it is needed to solve or calibrate 
the image Jacobi matrix in the practical application, according to the camera's internal and external parameters and the kinematic parameters of the robot [7].

In literature [8], a new eye-in-hand binocular stereo visual servoing model that the depth information can be ignored is proposed. In the new model, three image coordinate variables are introduced and which counteract the depth coordinate variable, so it is not needed to measure or estimate the depth information about the target. It is introduced for the target locating and tracking, and improves the control performance $[9,10,11]$. However, the model requires a strict calibration of the binocular cameras in addition to the image matching problem, which makes the system design become more complex. So it makes fast developments of the uncalibrated methods [12]. In recent years, the Kalman filter algorithm is widely used in the online estimation of the image Jacobi matrix. In literature [13] a Kalman filtering-based estimation algorithm is proposed to estimate the composite image Jacobian or the homogeneous transformation matrix on-line, which can reduce the influence of noise. Literature [14] designs an adaptive kalman filter to estimate the image Jacobi matrix on-line in the uncalibrated eye-in-hand systems. Literature [15] presents a Jacobi matrix on-line identification algorithm based on modified Kalman filter. The direct estimation of Jacobi matrix is realized by using the motion of the robot which simply improves the application of Kalman filter algorithm and makes up for the error caused by multiple iterations.

The Kalman filter has received a great success for its wide applicability and recursive algorithm. For stochastic linear systems, when the model is accurate and the statistics of process noise and measurement noise are Gauss white noise sequence with known variance, the Kalman filter has a perfect effect to estimate the state. But the robot control system in actual work will inevitably be affected by random noise, and when the statistics of the noise is not completely known, the filtering performance of the conventional Kalman filter will be reduced or even cause divergence [16]. The Cubature Kalman filter $(\mathrm{CKF})$ is a new nonlinear filtering method, which combines the radial integral rule and the spherical integral rule to achieve the accuracy of third order to high dimensional integral function (compared with the conventional Kalman filter). At the same time, it can be extended into the singular value decomposition aided Cubature Kalman filter to avoid matrix decomposition and inverse calculation, and improve the filtering stability [17].

In this paper, an uncalibrated binocular stereo visual servoing system using the new model in literature [8] is presented. According to analysis of its characteristics, the Jacobi matrix is estimated by the singular value decomposition aided Cubature Kalman Filter. To solve the problem that the unknown statistics of process noise and measurement noise will lead to low performance or divergence of Kalman filter, a neural network is added to the SVDCKF as the noise compensator which compensates the process noise and measurement noise. In order to verify the effectiveness of the algorithm, this paper establishes a simulink model of uncalibrated IBVS system based on the 6DOF puma560 robot using the robotics toolbox for MATLAB [18]. The image Jacobi matrix is respectively estimated by the three kinds of algorithms KF, SVDCKF, NNSVDCKF in unknown statistics of colored noise conditions with comparison and analysis.

\section{Formulation of Estimation Model for Image Jacobi Matrix}

\subsection{Binocular Stereo Visual Model}

Binocular stereo visual model in this paper is set to be eye-in-hand mode. The parameters of two cameras are the same and assume that their focal lengths $f$ is equal to the object distance. As seen in Figure 1, the origin $O$ of world coordinate coincides with the optical center $o 1$ of the left camera. The right camera with the optical center $o 2$ is parallel to the left one, and the length between the two lenses is $B$. In order to meet the people's habits, the image plane is in front of the camera center. We also define that the 
left image planes and right image plane are $x 1-y$ and $x 2-y$ respectively. Note that, as the two lenses is parallel to the installation, the displacement of the $Y$ coordinates in the two lenses is not much, so it is assumed that the vertical coordinate $y 1$ of image point in the left image is equal to $y 2$ in the right image, represented by y in this paper. Then according to the geometric relations in Figure 1, we can obtain the coordinates of image feature points $p \mathrm{~L}(x 1, \mathrm{y})$ and $p \mathrm{R}(x 2, \mathrm{y})$ in the left and right image plane respectively for the object point $\mathrm{P}(X, Y, Z)$.

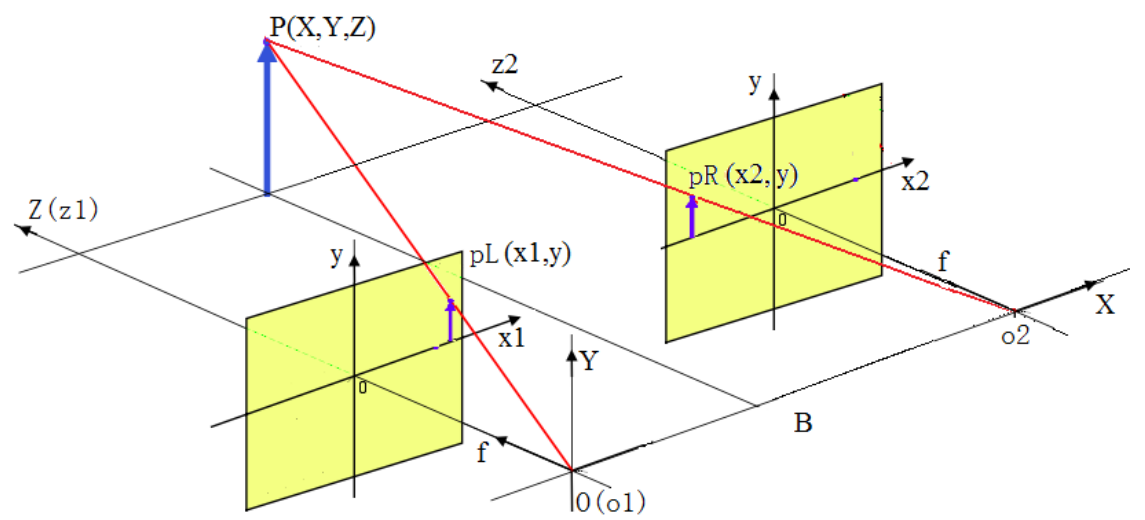

Figure 1. Binocular Stereo Visual Model

$$
\begin{gathered}
x 1=\frac{f}{Z} X \\
y=\frac{f}{Z} Y \\
x 2=\frac{-f}{Z}(B-X)
\end{gathered}
$$

Depth information $\mathrm{Z}$ can be obtained from the upper equations (1), (2) and (3)

$$
Z=\frac{f B}{x 1-x 2}
$$

Define ${ }^{C} P=[X, Y, Z]^{T}$ as the position coordinate matrix of object point, $C S=\left[S_{X C}, S_{Y C}, S_{Z C}\right]^{T}$ as the position coordinate matrix of the origin of the object point coordinate, and $c \theta=\left[\theta_{X C}, \theta_{Y C}, \theta_{Z C}\right]^{T}$ as the space angle matrix of the origin of the object point coordinate, then their relationships will be set up in (5) or expressed as (6)-(8).

$$
\begin{aligned}
{ }^{C} \dot{P} & ={ }^{C} \dot{S}+{ }^{C} \dot{\theta} \times{ }^{C} P \\
\frac{d X}{d t} & =\frac{d S_{X C}}{d t}+Z \frac{d \theta_{Y C}}{d t}-Y \frac{d \theta_{Z C}}{d t} \\
\frac{d Y}{d t} & =\frac{d S_{Y C}}{d t}-Z \frac{d \theta_{X C}}{d t}+X \frac{d \theta_{Z C}}{d t} \\
\frac{d Z}{d t} & =\frac{d S_{Z C}}{d t}+Y \frac{d \theta_{X C}}{d t}-X \frac{d \theta_{Y C}}{d t}
\end{aligned}
$$

For the eye-in-hand system, the end-effecter has relative motion to the object point, so the relationship of their motions is established as follow.

$$
\begin{gathered}
{ }^{T} S=\left[S_{X}, S_{Y}, S_{Z}\right]^{T}=-C S \\
{ }^{T} \theta=\left[\theta_{X}, \theta_{Y}, \theta_{Z}\right]^{T}=-C \theta
\end{gathered}
$$


where ${ }^{T} S=\left[S_{X}, S_{Y}, S_{Z}\right]^{T}$ is the position coordinates matrix of the origin of the endeffecter coordinate of robot, and ${ }^{T} \theta=\left[\theta_{X}, \theta_{Y}, \theta_{Z}\right]^{T}$ is the space angle matrix of the origin of the end-effecter coordinate of robot.

$$
\left[\begin{array}{l}
\dot{x} 1 \\
\dot{y} \\
\dot{x} 2
\end{array}\right]=\left[\begin{array}{cccccc}
-\frac{f}{Z} & 0 & +\frac{x 1}{Z} & +\frac{x 1 y}{f} & -\frac{f^{2}+x 1^{2}}{f} & +y \\
0 & -\frac{f}{Z} & +\frac{y}{Z} & +\frac{f^{2}+y^{2}}{f} & -\frac{x 1 y}{f} & -x 1 \\
-\frac{f}{Z} & 0 & \frac{x 1}{Z}-\frac{f B}{Z^{2}} & \frac{x 1 y}{f}-\frac{B}{Z} y & -\frac{f^{2}+x 1^{2}}{f}+\frac{B}{Z} x 1 & +y
\end{array}\right] \cdot\left[\begin{array}{c}
\dot{S}_{X} \\
\dot{S}_{Y} \\
\dot{S_{Z}} \\
\dot{\theta}_{X} \\
\dot{\theta}_{Y} \\
\dot{\theta}_{Z}
\end{array}\right]
$$

Substituting (4) to (11), the relationship without depth information $\mathrm{Z}$ can be obtained.

$$
\left[\begin{array}{l}
\dot{x} 1 \\
\dot{y} \\
\dot{x} 2
\end{array}\right]=\left[\begin{array}{cccccc}
\frac{y-x 1}{B} & 0 & \frac{x 1(x 1-x 2)}{f B} & +\frac{x 1 y}{f} & -\frac{f^{2}+x 1^{2}}{f} & +y \\
0 & \frac{x 2-x 1}{B} & \frac{y(x 1-x 2)}{f B} & +\frac{f^{2}+y^{2}}{f} & -\frac{x 1 y}{f} & -x 1 \\
\frac{y-x 1}{B} & 0 & \frac{x 2(x 1-x 2)}{f B} & \frac{+y x 2}{f} & -\frac{f^{2}+x 1 x 2}{f} & +y
\end{array}\right] \cdot\left[\begin{array}{c}
\dot{S}_{X} \\
\dot{S}_{Y} \\
\dot{S}_{Z} \\
\dot{\theta}_{X} \\
\dot{\theta}_{Y} \\
\dot{\theta}_{Z}
\end{array}\right]
$$

Define $p_{\text {single }}=[x 1, y, x 2]^{T}$ as the position matrix of the image feature points for single object point in the left and right image, $U=\left[S_{X}, S_{Y}, S_{Z}, \theta_{X}, \theta_{Y}, \theta_{Z}\right]^{T}$ as the position and pose matrix of the end-effecter in world coordinate, (12) can be expressed as

$$
\dot{p}_{\text {sin } g l e}=J\left(p_{\text {sin } g l e}\right) \dot{U}
$$

where $J\left(p_{\text {single }}\right)$ is the image Jacobi matrix for the feature point of single object point.

Compared to monocular vision model, the proposed Jacobi matrix of the binocular vision model in (12) or (13) do not contain the depth information $\mathrm{Z}$, which will simplify the design of the IBVS system. If the object is not represented as single point, and has more than one image feature point such as m points, (13) can be extended to form for $m$ feature points as follow

$$
\dot{p}=\left[\begin{array}{l}
\dot{p}_{\text {sin } g l e, 1} \\
\dot{p}_{\text {sin } g l e, 2} \\
\cdots \\
\dot{p}_{\text {sin } g l e, m}
\end{array}\right]=\left[\begin{array}{l}
J_{\text {single, } 1}\left(p_{\text {single, } 1}\right) \\
J_{\text {single, } 2}\left(p_{\text {sin } g l e, 2}\right) \\
\cdots \\
J_{\text {sin } g l e, m}\left(p_{\text {sin } g l e, m}\right)
\end{array}\right] \dot{U}=J \dot{U}
$$

where $p_{3 m \times 1}$ is the position matrix of the $m$ image feature points for object in the left and right image, $J_{3 m \times 6}$ is the image Jacobi matrix, $p_{\text {single,i }}=\left[x 1_{i}, y_{i}, x 2_{i}\right]^{T}$ is the position matrix of the $i$-th image feature points for object in the left and right image, and $J_{\sin g l e, i}$ is the corresponding $i$-th image Jacobi matrix, $i=1,2,3, \ldots . . m$.

\subsection{Estimation Model for Image Jacobi Matrix}

If we want to estimate the image Jacobian matrix using Kalman filter algorithm, it is needed to establish the process equation and measurement equation with the state vector composed of elements of image Jacobian matrix. The process is as follows.

Discretize (14), and get 
$\left(p_{k+1}-p_{k}\right) / \Delta t=J \cdot \Delta U_{k} / \Delta t$

where $\Delta U_{k}$ is the changes of as the position and pose matrix of the end-effecter between instant $k-1$ and $k$.

Construct the state vector $\chi_{k}$ in each element of image Jacobi matrix, and measurement vector $Z_{k}$, as $\chi_{k}=\left[J_{1,1}, J_{1,2}, \ldots J_{1,6}, J_{2,1}, \ldots J_{m, 6}\right]_{18 m \times 1}^{T}$ and $Z_{k}=\left[p_{k+1}-p_{k}\right]_{3 m \times 1}$.

Then the linear discrete dynamic system is established in process equation (16) and measurement equation (17)

$$
\begin{gathered}
\chi_{k+1}=\chi_{k}+\omega \\
Z_{k}=H_{k} \cdot \chi_{k}+v
\end{gathered}
$$

where $\omega_{18 m \times 1}$ represents process noise vector which is uncorrelated zero-mean Gaussian white noise sequences with covariance $Q_{18 m \times 18 m}$ and $v_{3 m \times 1}$ measurement one with covariance $R_{3 m \times 3 m}$ defined the same as $\omega . H_{k}$ is the measurement matrix, obtained as

$$
H_{k}=\left[\begin{array}{ccc}
\Delta \mathrm{U}_{\mathrm{k}}{ }^{\prime} & \cdots & 0 \\
\cdots & \Delta \mathrm{U}_{\mathrm{k}}{ }^{\prime} & \cdots \\
0 & \cdots & \Delta \mathrm{U}_{\mathrm{k}}^{\prime}
\end{array}\right]_{3 m^{*} 18 m}
$$

\section{NNSVDCKF for Jacobi Estimation}

\subsection{SVDCKF Algorithm}

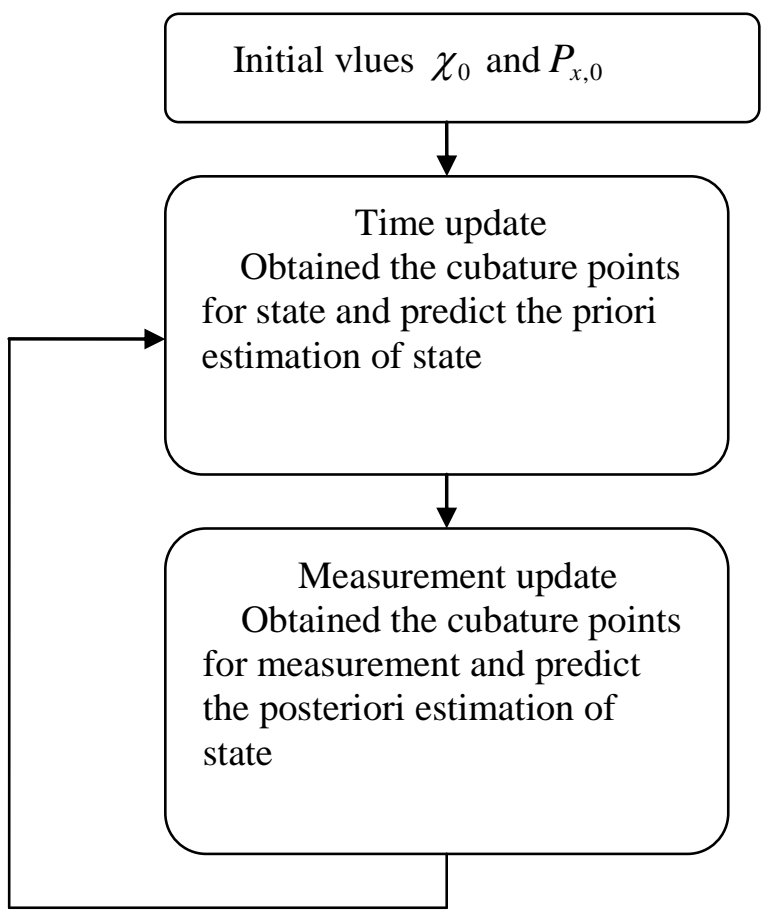

Figure 2. The Structure of CKF

The basic structure of CKF is consistent with other Kalman filters (KF, UKF), which is composed of two parts, the time update and measurement update shown in Figure 2. However, the accuracy of CKF algorithm is higher than that of KF and UKF on the 
premise of equivalent computing complexity. In the standard CKF algorithm, Cholesky decomposition is used for obtaining cubature points. In the actual filtering calculation, because of the truncation error of computer, the error covariance matrix $\mathrm{P}$ is often lost its positive definiteness and symmetry. This case will be further deteriorated during inverting and decomposing matrix in $\mathrm{CKF}$, and lead to filtering instability and even divergence.

Singular value decomposition is a best matrix decomposition form of numerical stability and accuracy in numerical algebra so far, and is easy to be implemented on vector parallel computers. So that using the singular value decomposition to replace Cholesky decomposition can improve the stability of the CKF algorithm, as follows:

Taking the square root of covariance matrix $P_{x, k}$ by SVD

$$
P_{x, k}=U\left[\begin{array}{cc}
S & 0 \\
0 & 0
\end{array}\right] V^{T}
$$

where $S$ is a diagonal matrix, $S=\operatorname{diag}\left[\begin{array}{llll}s_{1} & s_{2} & \ldots & s_{L}\end{array}\right]$. In general, the covariance matrix $P_{x, k}$ is symmetric, so $U=V$. The characteristic values are $s_{i}^{2}(\mathrm{i}=1,2, \ldots, \mathrm{L})$, and the feature vector of the $P_{x, k}$ represented by column vectors of $U U^{T}$. The cubature points are calculated as follow

$$
X_{i, k}=U \sqrt{S} \xi_{i}+\hat{\chi}_{k}^{+} \quad i=1,2 . .2 n
$$

The complete process of SVDCKF algorithm can be seen in literature [19].

\subsection{Noise Compensator Based On Neural Network}

There are two main factors affecting the accuracy of the estimation while using Kalman filter. One is process error $w$ with covariance $Q$, and the other is the measurement error $v$ with covariance $R$. The Kalman filter algorithm is designed to achieve the minimum variance estimation of a linear stochastic system under the assumption that the process noise and observation noise are the Gauss white noise. But in the actual system, the statistics information of the two kinds of noises are difficult to obtain, and may be non Gauss colored noise at most time. If the filter in the dynamic data processing is too dependent on the error model, it is easy to lead to system deviation on the basis of the error accumulation of filtering results and even filtering divergence. Therefore, we need to introduce adaptive algorithm to compensate the $Q$ and $R$ in the estimation process. If the uncertainty of the system model is high, and the measurement information is relatively accurate, it is required to reduce the $Q$, increase $R$. Vice versa.

In this paper, we put forward using neural network as a noise compensator which compensate the system noise and measurement noise covariance $Q$ and $R$ before each filtering process. The neural network is designed as two-layer BP neural network, and its topology structure as shown in Figure 3.

A. input layer.

The put matrix of the BP neural network is $x=\left[\begin{array}{llll}x_{1} \ldots & x_{j} & \ldots & x_{m n+m}\end{array}\right]$, where $x_{j}$ represents the input of $j$-th node in the input layer $j=1, \ldots, m n+n$.

$x$ contains two types of input signal.

1) The state estimation error between the current instant $\mathrm{k}$ and instant $k-1$, as

$\Delta e_{\chi}=\hat{\chi}_{k}-\hat{\chi}_{k-1}$

2) The innovation at instant $k$, as

$$
V_{k}=Z_{k}-H_{k} \hat{\chi}_{k-1}
$$




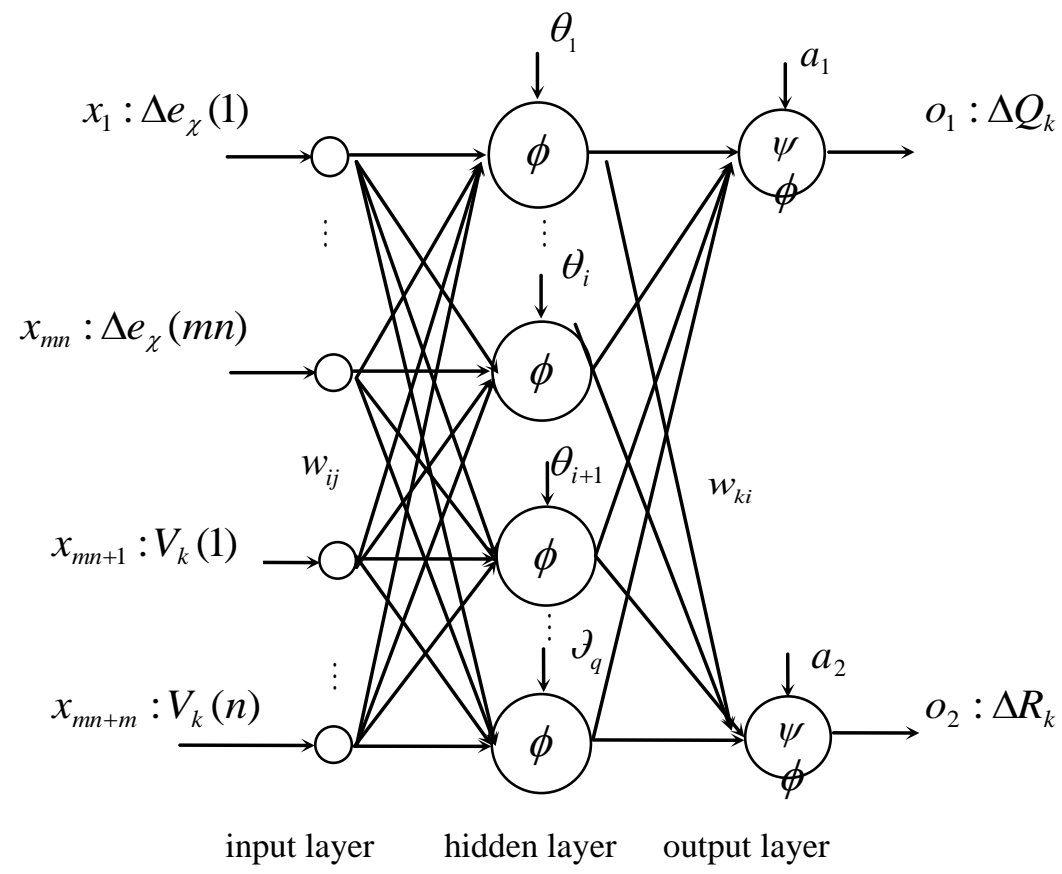

Figure 3. Topology Structure of BP Neual Network

B. Hidden layer.

The output $y_{i}$ of the $i$-th node in the hidden layer is defined as

$y_{i}=\phi\left(\right.$ net $\left._{i}\right)=\phi\left(\sum_{j=1}^{M} w_{i j} x_{j}+\theta_{i}\right)$

where $w_{i j}$ is the weights from the $i$-th node in hidden layer to the $j$-th node in input layer, $\theta_{i}$ is the threshold of the $i$-th node in hidden layer, and $\phi(\cdot)$ is the excitation function in hidden layer.

C. Output layer

The output $o_{l}$ of the $l$-th node of the output layer is

$o_{l}=\psi\left(\right.$ net $\left._{l}\right)=\psi\left(\sum_{i=1}^{q} w_{l i} y_{i}+a_{l}\right)=\psi\left(\sum_{i=1}^{q} w_{l i} \phi\left(\sum_{j=1}^{M} w_{i j} x_{j}+\theta_{i}+a_{l}\right)\right.$

where $w_{l i}$ is weights from the $l$-th node in the output layer to the $i$-th node in hidden layer, $i=1, \ldots, q ; a_{l}$ is the threshold of the $l$-th node in the output layer, $l=1,2$; and $\psi(x)$ is the excitation function of the output layer.

Furthermore, the output $o_{l}$ can be defined as $o_{1}=\Delta Q_{k}$ and $o_{2}=\Delta R_{k}$, representing the compensated value of $Q$ and $R$ at instant $k$ respectively.

$D$. The total error criterion function for $\mathrm{P}$ training samples.

$E=\frac{1}{2} \sum_{p=1}^{P} \sum_{l=1}^{2}\left(T_{l}^{p}-o_{l}^{p}\right)$

According to error gradient descent method, the weight corrections $\Delta w_{l i}$ and the threshold corrections $\Delta a_{l}$ of the output layer, as well as the weight corrections $\Delta w_{i j}$ and the threshold corrections $\Delta \theta_{i}$ of the output layer are obtained as follow. 
$\Delta w_{l i}=-\eta \frac{\partial E}{\partial w_{l i}}, \Delta a_{l}=-\eta \frac{\partial E}{\partial a_{l}}, \Delta w_{i j}=-\eta \frac{\partial E}{\partial w_{i j}}, \Delta \theta_{i}=-\eta \frac{\partial E}{\partial \theta_{i}}$

where $\eta$ is the learning rate (or termed momentum factor), and taken about 0.1 to 0.4 .

In this paper, Sage-husa filtering method [20] is used to obtain input-output sample pairs for training the neural network.

First estimate the observation noise covariance matrix.

$\hat{R}_{k}=\hat{C}_{v k}-H_{k} P_{k}^{-} H_{k}^{T}$

where $P_{k}^{-}$is the priori covariance matrix, $C_{v k}$ is the covariance of innovation, and $\hat{C}_{v k}$ is the estimation of $C_{v k}$ obtained as

$\hat{C}_{v k}=\frac{1}{N} \sum_{j=0}^{N-1} V_{k-j} V_{k-j}^{T}$

Second, estimate the process noise covariance matrix.

$\hat{Q}_{k-1}=E\left\{w_{k-1} w_{k-1}^{T}\right\}=K_{k} \hat{C}_{v k} K_{k}^{T}$

where $K_{k}$ is the kalman filter gain.

Finally, get the new values of the $Q$ and $R$ applied to the kalman filter at instant $k$.

$Q_{k}(i, i)=Q_{k-1}(i, i)+\Delta Q_{k}, \quad i=1,2, \ldots, m n$

$R_{k}(i, i)=R_{k-1}(i, i)+\Delta R_{k}, \quad i=1,2, \ldots, n$

where

$$
\begin{gathered}
\Delta Q_{k}=\operatorname{mean}\left(\sum_{i=1}^{m n}\left(\hat{Q}_{k-1}(i, i)-Q_{k-1}(i, i)\right)\right) \\
\Delta R_{k}=\operatorname{mean}\left(\sum_{i=1}^{n}\left(\hat{R}_{k-1}(i, i)-R_{k-1}(i, i)\right)\right)
\end{gathered}
$$

\subsection{Uncalibrated Binocular Stereo Visual Servoing System}

The structure of proposed uncalibrated binocular stereo visual servoing system, which controls the end-effecter moving to the specified location, is shown in Figure 4.

Define $K$ as a positive definite matrix, and the controller is as follows:

$u=J(p)+K e=J(p)+K\left(p_{d}-p\right)=\mathrm{U} / \Delta \mathrm{T}$

where $p_{d}$ is the current position, $p$ is desired location, and $e$ is image error of the image feature point; $J(p)^{+}$is the pseudo-inverse matrix of $J(p)$

As shown in Figure 4, The SVDCKF is to estimate the image Jacobi matrix, and the neural network is to compensate $Q$ and $R$ in the SVDCKF. Firstly, the Kalman filter is initialized by $\hat{X}_{k}^{+}, P_{0}, Q_{0}$, and $R_{0}$. According to the difference between desired and actual location of the feature points in the image plane, the pose and position variation $Z(k)$ of the end-effecter can be obtained, as well as the observation matrix $H(k)$. Then, estimate the image Jacobi matrix using SVDCKF. At the same time, also get the $\Delta Q_{k}$ and $\Delta R_{k}$ from the $\mathrm{NN}$, which will be fed back to the SVDCKF. Finally, the pseudo-inverse matrix of estimated of $J(p)$ can be used for the controller in (34) to obtain the control amount for each joint of the robot, moving the end-effecter to the desired location. The iteration loop will end, if error is 0 or control time ends. 


\section{Simulation and Analysis for Uncalibrated Binocular Stereo Servoing System}

\subsection{Establishment of Simulation Model}

To verify the effectiveness of proposed algorithm, the paper designs a uncalibrated binocular stereo servoing system based on PUMA560 using the binocular vision model presented in section2. The distance between optical centers of the two cameras is $10 \mathrm{~cm}$. And the camera parameters are the same, as follows: the effective focal length is $8 \mathrm{~mm}$, the sensitive array is $6.4 \mathrm{~mm} \times 6.4 \mathrm{~mm}$ with $512 \times 512$ pixels, and each pixel is corresponding to the image size of $0.0125 \mathrm{~mm}^{2}$. The origin in the image plane is [256, 256].

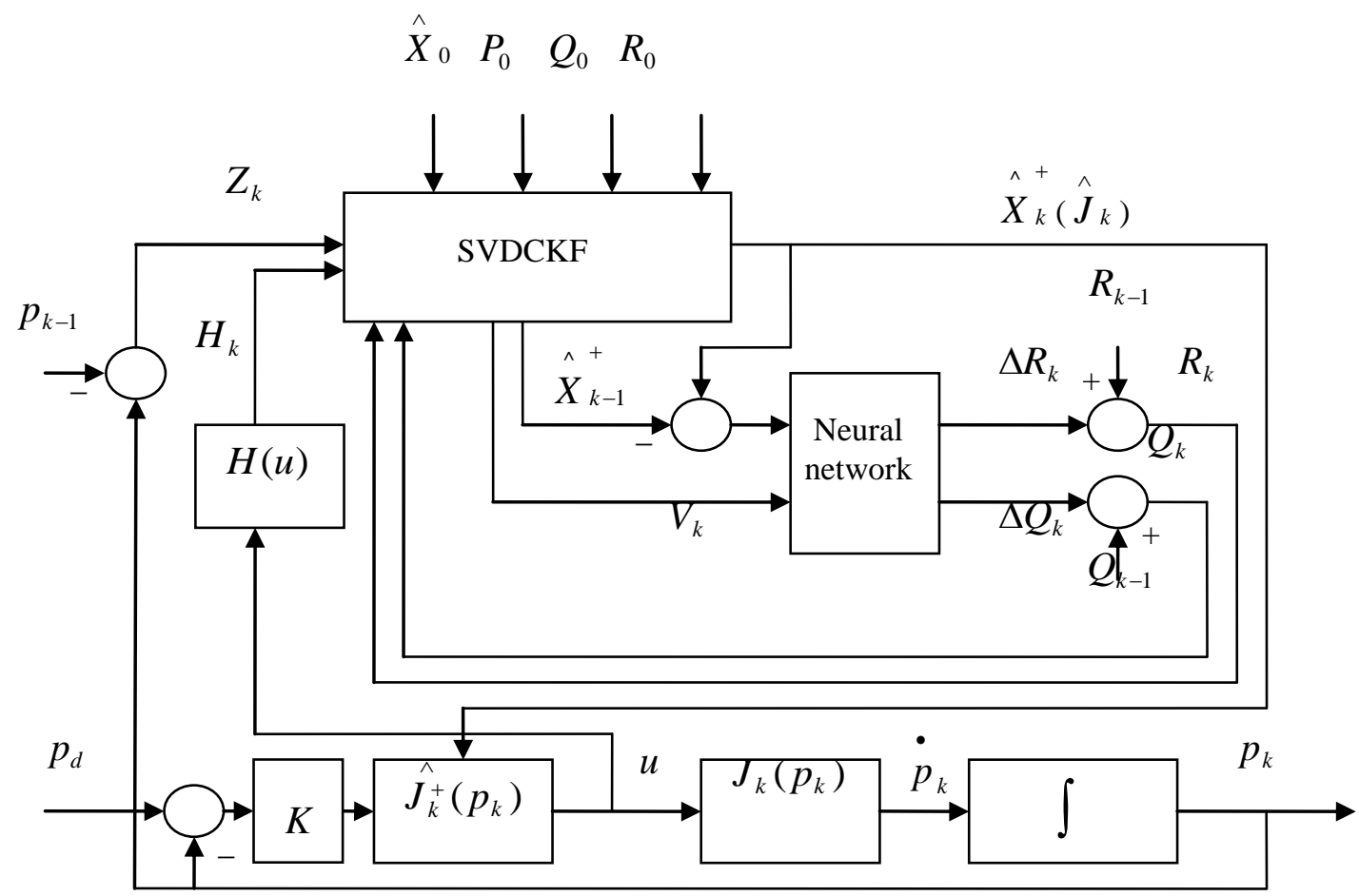

Figure 4. The Structure of Uncalibrated Binocular Stereo Visual Servoing System 


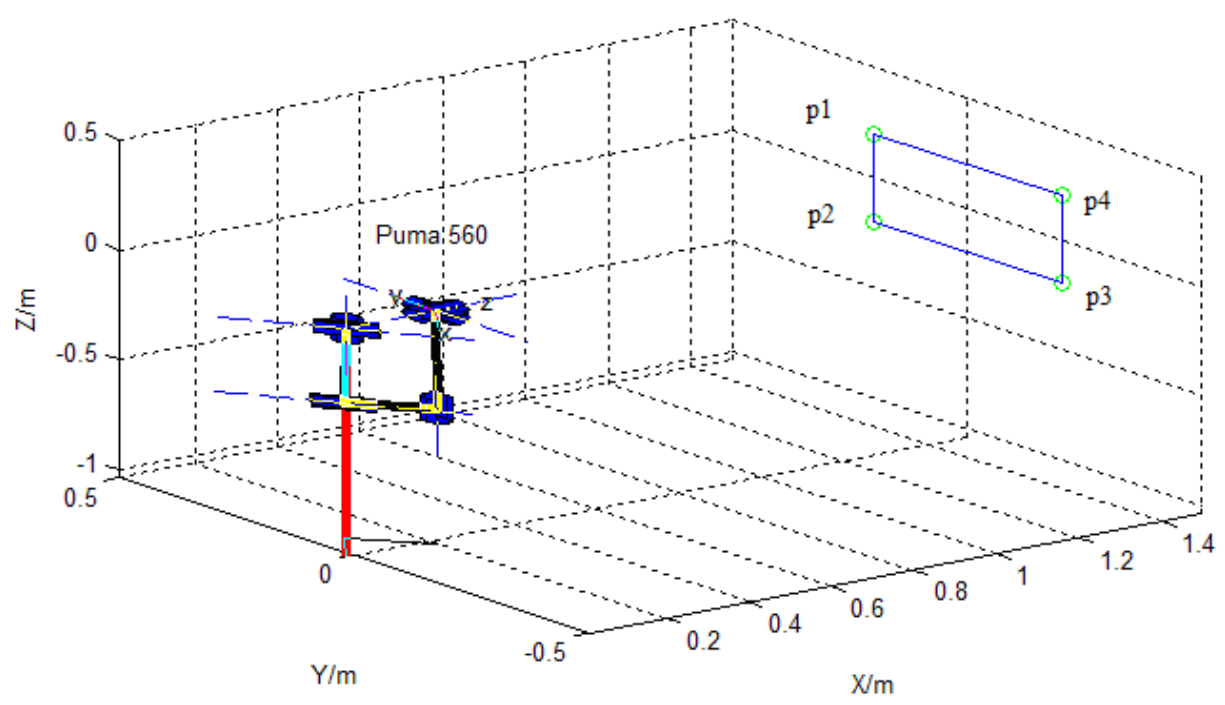

Figure 5. The Object and PUMA560

The right hand coordinate system of the visual system and generalized focal length model are applied in this simulation using pixel coordinates. The object is a rectangular with four vertices as feature points, $\mathrm{p} 1(1.5,0.2,0.2)$, p2 $(1.5,0.2,-0.2), \mathrm{p} 3(1.5,-0.2$, $0.2)$ and $\mathrm{p} 4(1.5,-0.2,0.2)$, and the unit is $\mathrm{m}$. The initial angle of six axis of PUMA560 manipulator is set as $[0.7726,1.3185,1.2872,0.832,1.2872,1.3185]$, and the initial position of its end-effecter is [0.206, 0.009, 0.206], as shown in Figure 5.

Based on the above set for IBVS, the simulation model of uncalibrated binocular visual servoing control system is established using the robotics toolbox for MATLAB as shown in the Figure 6.

The differences of the expected and actual position for feature points in left and right image planes and changes of controlled variable are given as inputs for the function NN_SVDCKF. Utilizing the proposed filtering algorithm, the estimated image jacobi matrix which is the output of NN_SVDCKF, is obtained for calculating the control amount of the end-effecter, which make up the whole servoing closed-loop.

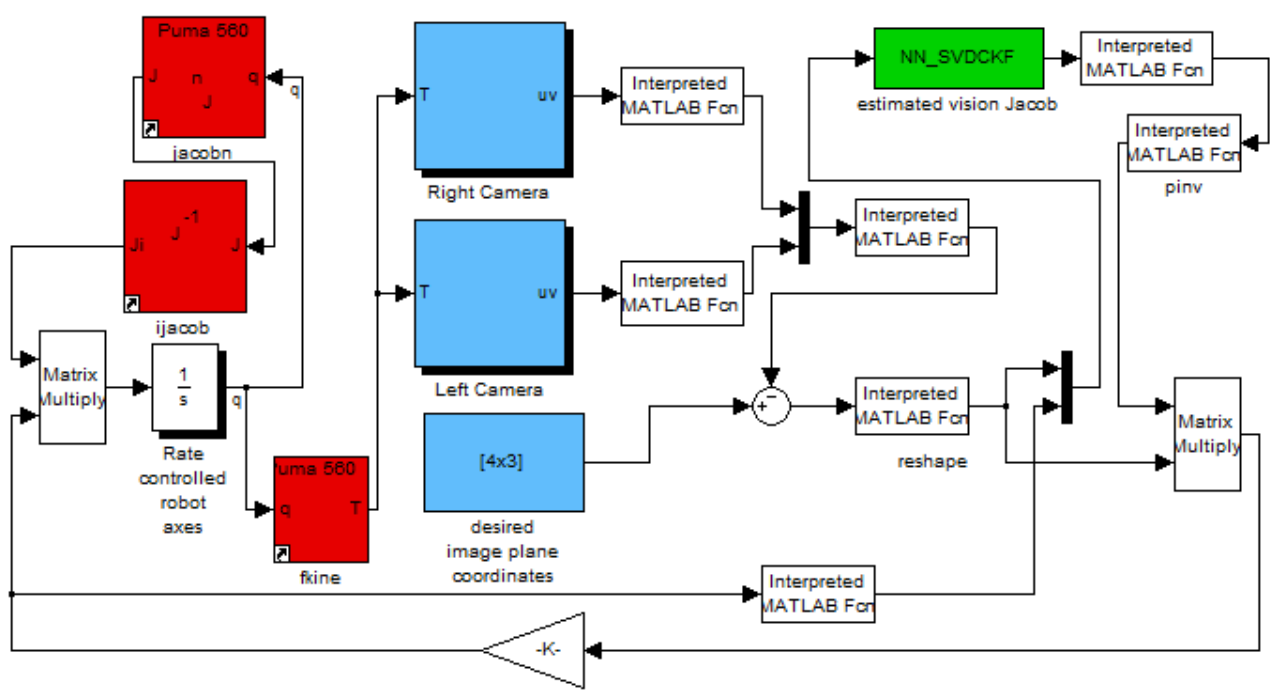

Figure 6. Simulation Model of Uncalibrated Binocular Visual Servoing Control System 


\subsection{Simulation and Analysis}

Before simulation of the system, this paper obtains 70 pairs of the input-output sampling data to train neural network using the method given in section3.3 by KF. The hidden layer contains 100 neurons, and the training reaches the best validation performances having the minimum sum squared error (MSE) is $1.08 \times 10^{-18}$. It is seen that the test output of the network is very close to the training of the output.

The initial position $p$ of the four feature points in image plane defined in section 2 can be represented as $p=\left[\begin{array}{llllll}203.4469 & 396.5352 & 154.3883 & 404.5692 & 399.0493 & 353.5409 \text {; }\end{array}\right.$ $402.3523197 .8728197 .8728 ; 204.8015199 .2643$ 156.5931], and its corresponding expected position pd is set $p_{d}=[100,400,25,100,400,325,100,100,325,100,100$, 25], with unit for the pixel. The controller gain is $K=0.5 I$, where $I$ is the unit matrix with appropriate dimension.

To verify the robustness of NNSVDCKF algorithm to the unknown statistics of noises, colored noises are given to the system for estimation of Jacobi. That is, $Q \in N(20,20)$, and $R \in N(5,3)$. The covariance matrix $P_{0}=0.1 I$. Then, under the situation of colored noises, comparison end analysis are made among the three algorithms KF, SVDCKF and NNSVDCKF with initial white Gaussian noise $Q \in N(0,20)$ and $R \in N(0,3)$.

Figure 7 gives the trajectory curves of feature points and end-effecter. The first and second columns in Figure 7 are trajectory curves of feature points in left and right image planes using KF, SVDCKF and NNSVDCKF respectively, while the third one is the corresponding trajectory of the end-effecter. It is not hard to find that, though all the three algorithms can make the feature points moving to the desired location in image plane, but the trajectories of feature points are different each other. Take the third feature point for example, servoing system using NNSVDCKF has the most gentle trajectories which are the least influenced by colored noise compared to SVDCKF and KF. It also be demonstrated in the third column of Figure 7, that the trajectory of end-effecter has the shortest movement using NNSVDCKF.

At the same time, it can also be found that the proposed method has a better robustness for boundary constraints of FOV of the left and right camera. In the simulation, the desired location of feature points is on the left of the initial position. The black lines in the first and second column of Figure 7 is used to represent the upper and right border of trajectory, from which it is can be confirmed that if the initial position of feature points is very close to the boundary in left image plane, the trajectory using KF or SVDCKF may more easily get out of the FOV of cameras than that using NNSVDCKF. 

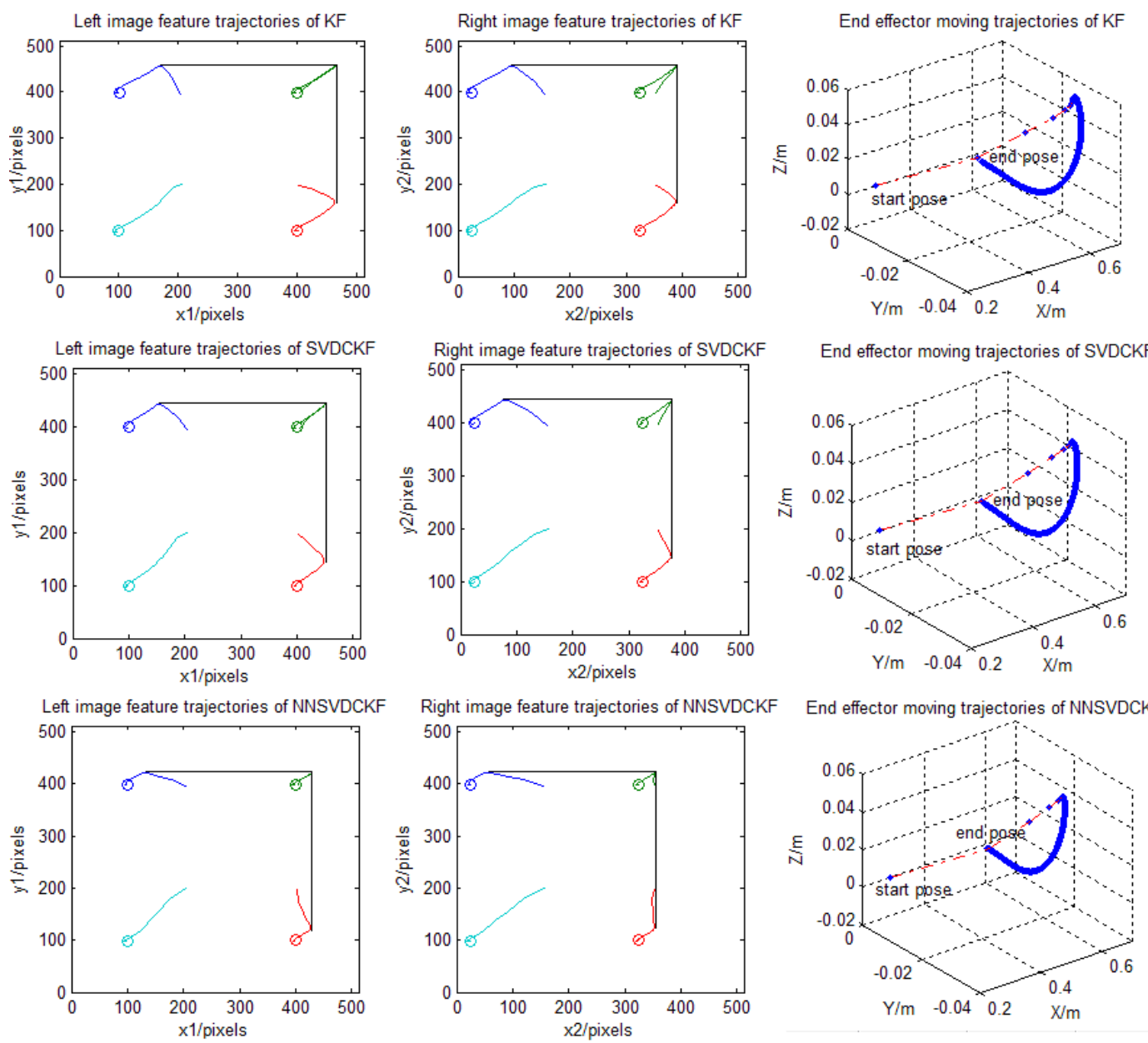

Right image feature trajectories of NNSVDCKF

End effector moving trajectories of NNSVDCKF
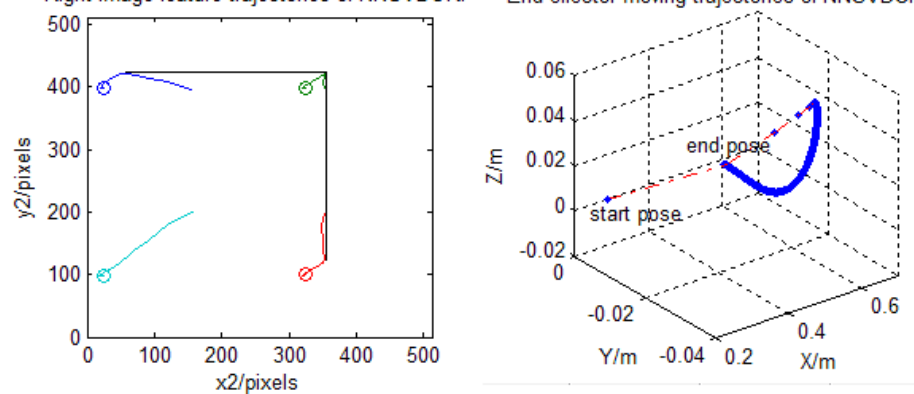

\section{Figure 7. The Trajectory Curves of Feature Points and End-effecter Using KF, SVDCKF and NNSVDCKF}

The image feature errors of third feature point in left image plane are given in Figure 8. The errors of $x 1$ using KF in the initial phase is nearly three times and twice to that by NNSCDCKF and SVDCKF respectively. It can also be find the settling time when the errors approximates 0 is the shortest for servoing system using NNSVDCKF no matter errors of $x 1$ or errors of $y 1$. 


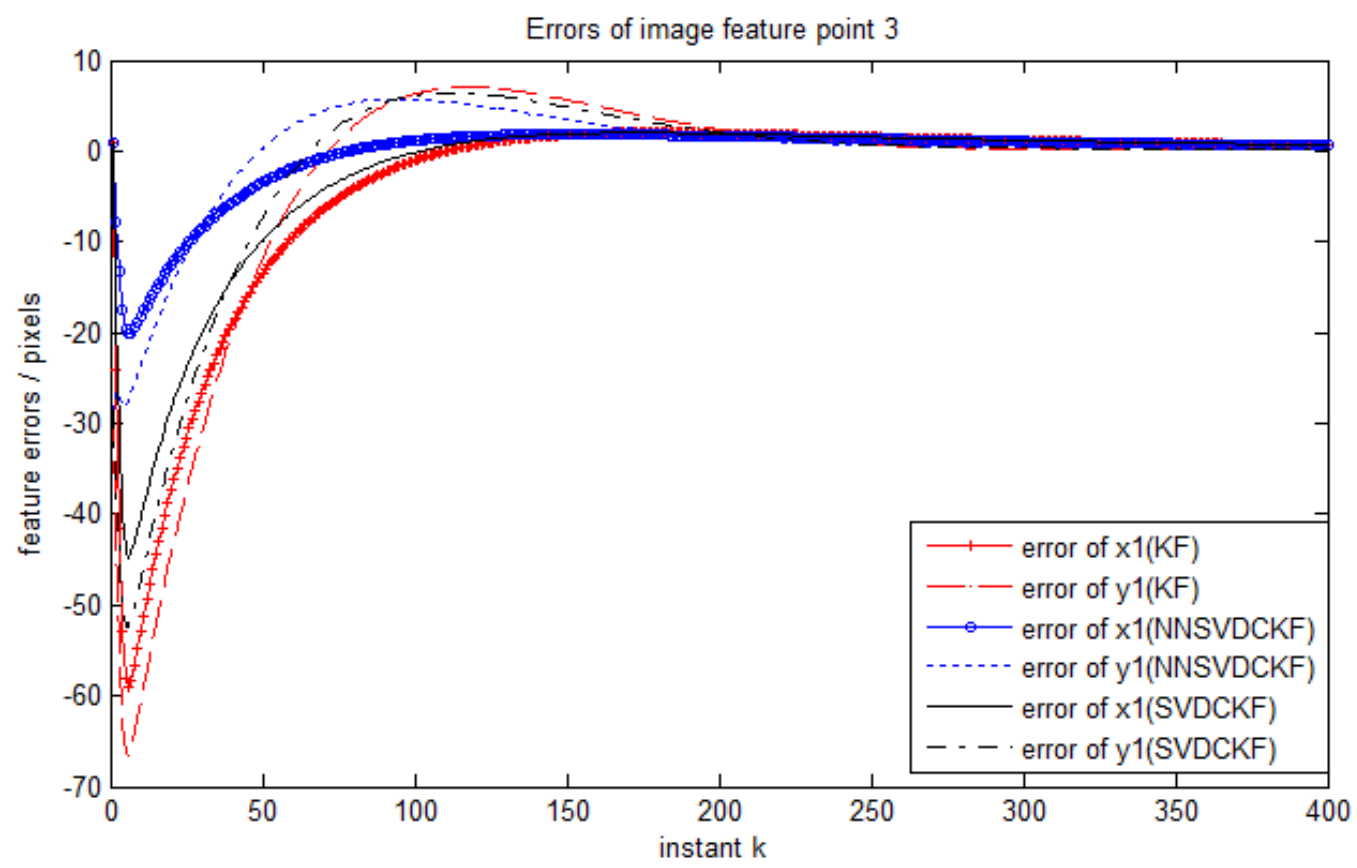

Figure 8. The Image Feature Errors of Third Feature Point in Left Image Plane

\section{Conclusion}

The prominent advantage of binocular vision servoing system compared with monocular vision system is that it does not need the depth information. However in actual work, its accuracy of estimation of image Jacobian matrix for uncalibrated visual servoing control system has also been influenced by unknown statistics of process noise and measurement noise. This paper not only uses singular value decomposition aided CKF to estimate the Jacobi for higher accuracy compared with kalman filtering, but also compensate for the unknown noises by a neural network. Simulation and analysis are made among the KF, SVDCKF and NNSVDCKF in the case of colored noises with unknown statistics. Simulation results show that NNSVDCKF algorithm can improve the dynamic performance of the servoing system, and also has better robustness to constraint in FOV of the camera.

\section{Acknowledgments}

This work was supported by National Natural Science Foundation of Hebei Province (No.F2012203111) and Natural Science Youth Foundation of Higher Education of Hebei Province (No. 2011139).

\section{References}

[1] S. Hutchinson, G. Hager and P. Corke, "A tutorial on visual servo control", IEEE Transactions on Robotics and Automation, vol. 12, no. 5, (1996), pp. 651-670.

[2] L. Qeiss, A. C. Sanderson and C. Neuman, "Dynamic sensor-based control of robots with visual feedback", IEEE Journal of Rohotics and Automation, vol. 3, no. 5, (1987), pp. 404-417.

[3] P. Jorge, Sensors Pablo and T. Pernando, "Visual control of robots using range images", Sensors, no.10, (2010), pp. 7303-7322.

[4] S. Segvic, A. Remazeilles and F. Chaumette, "Experimental evaluation of autonomous driving based on visual memory and image-based visual servoing", IEEE Transactions on Intelligent Transportation Systems, vol. 12, no.3, (2011), pp.870-883. 
[5] X.G. Zhong, X.Y. Zhong and X.P. Peng, "Robust kalman filtering cooperated elman neural network learning for vision-sensing-based robotic manipulation with global stability", Sensors, no. 13, (2013), pp. 13464-13486.

[6] S.S. Mehta, V. Jayaraman, T.F. Burks and W.E. Dixon, "Teach by zooming: A unified approach to visual servo control", Mechatronics, vol. 22, no. 3, (2012), pp. 436-443.

[7] H. N. Nguyen,J. Zhou and H. J. Kang, "A calibration method for enhancing robot accuracy through integration of an extended Kalman filter algorithm and an artificial neural network", Neurocomputing, no. 151, (2015), pp. 996-1005.

[8] H. G. Li, Z. B. Kang, C. H. Leng, M. Jin and L. Y. Zou, "A new model of binocular vision in imagebased visual servoing robot control", Journal of Yanshan University, vol. 35, no. 3, (2011), pp. 215-222.

[9] X. P. Sha,H. G. ,Li, C. Wen and F. Li, "Stereo microscopic visual servoing control based on the optical flow technology", Optical Engineering, vol 10, no. 23, (2014), pp. 10251-10258.

[10] B. Yang, H. G. Li, Z. B. Kang and H. L. Jiang, "Hamiltonian-based binocular visual servoing of camerain-hand robotic systems", Proceedings of 2012 International Conference on Modelling, Identification and Control, Wuhan, China, (2012) June 24-26.

[11] N. Shao, H. G. Li, L. Liu and G. Y Li, "Distributed cooperative control for multi-robot systems based on binocular visual”, ICIC Express Letters, vol. 9, no. 7, (2015), pp. 2051-2058.

[12] D.I. Kosmopoulos, "Robust Jacobian matrix estimation for image-based visual servoing", Robot Comput.-Integr. Manuf, vol. 27, (2011), pp. 82-87.

[13] J Wang, A Liu, X Tao and H Cho, "Microassembly of micropeg and -hole using uncalibrated visual servoing method", Precision Engineering, vol.32, no. 3, (2008), pp.173-181.

[14] A. J. Xin, L. Bai and D. Liu, "Adaptive Kalman Filter-based Robot 6DOF Uncalibrated Vision Positioning", Journal of System Simulation, vol. 26, no. 3, (2014), pp. 586-591.

[15] H. B. Wang and P Li, "on-line estimation for image jacobin matrix based on improved Kalman filter", Journal of wuhan university of technology, vol. 31, no.14, (2009), pp. 145-148.

[16] X. D. Lv and X. H. Huang, "Fuzzy Adaptive Kalman Filtering based Estimation of Image Jacobian for Uncalibrated Visual Servoing", International Conference on Intelligent Robots and Systems, Beijing, china, (2006) October 9-15.

[17] Arasaratnam and S. Haykin, "Cubature Kalman filters", IEEE Transactions on Automatic Control, vol. 54, no. 6, (2009), pp. 1254-1269.

[18] P. I. Corke, "Robotics Toolbox for MATLAB", Robotics \& Automation Magazine IEEE, vol. 3, no. 1, (1996), pp. 24-32.

[19] Q. Z. Zhang, S. B. Zhang,Z. P. Liu and H. F. Bian, "Robust cubature Kalman filter based on SVD and its application to integrated navigation", Control \& Decision, vol. 29, no. 2, (2014), pp. 341-346.

[20] L. Shang, G. H. Liu, R. Zhang and G. T. Li, "An Adaptive Kalman Filtering Algorithm for Autonomous Orbit Determination Based-on BP Neural Network", Journal of Astronautics, vol. 34, no. 7, (2013), pp. 926-931.
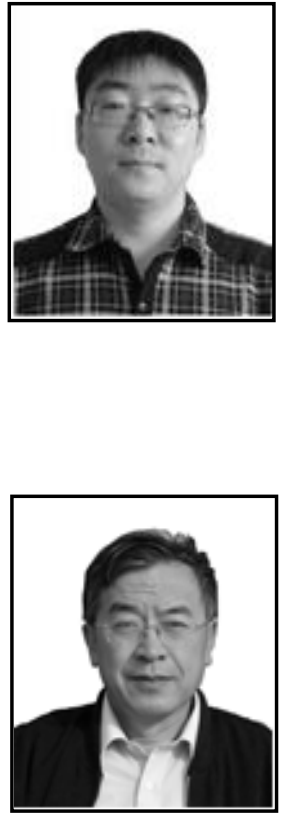

\section{Authors}

Wei Zhao, he was born in Hebei, China in 1980. He received his B.S. degree in automation from the Beihang University, Beijing, China in 2003, and M.S. degree in control theory and control engineering from Hebei polytechnic University, Hebei, China in 2008. He is currently a Ph.D. candidate in the School of Electrical Engineering, Yanshan University, Hebei, China. Since 2008, he has been with North China University of Science and Technology where he is currently an associate professor. His main research interests include industry automation, nonlinear control and robot visual servoing control.

Huiguang $\mathbf{L i}$, he is a professor at Yanshan University. He received his M.S. degree from Northeast Heavy Machinery Institute in 1982. He is the author of more than 30 journal papers and has written 4 books. His current research interests include robot visual servoing and control, sampling system theory, metallurgical industry automation and microcomputer control systems. 


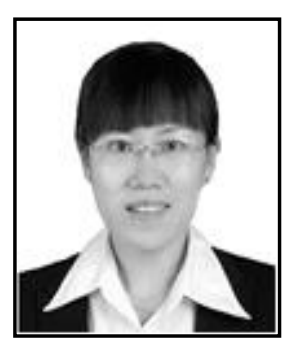

Liying Zou, she was born in 1980. She received her B.S. degree in automation, and the M.S. degree in control theory and control engineering from Qiqihar University in 2003, and Yanshan University in 2009, respectively, all in China. She is currently a Ph.D. candidate in the School of Electrical Engineering of Yanshan University, Hebei, China. Since 2003, she has been at the Department of Automation, Qiqihar University, Qiqihar, China. Her main research interests are centered on nonlinear control, aircraft control and visual servoing control.

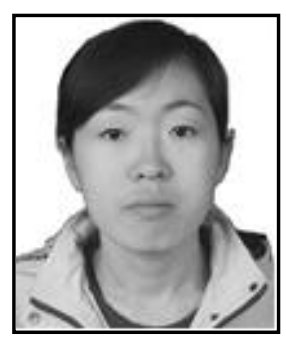

Wenjuan Huang, she was born in Hebei, China in 1981. She received her B.S. degree in Control Technology and Instrument Professional from the Hebei University, Baoding, China in 2005 and M.S. degree in control theory and control engineering from Hebei polytechnic University, Hebei, China in 2008. Since 2008 she has been at Tangshan Vocational and Technical College, Tangshan, China. Her main research interests are centered on nonlinear control, and metallurgical industry automation. 
International Journal of Control and Automation Vol. 9, No. 12 (2016) 